\title{
ATITUDES DOS ENFERMEIROS DE CENTRO CIRÚRGICO DIANTE DA SISTEMATIZAÇÃO DA ASSISTÊNCIA DE ENFERMAGEM PERIOPERATÓRIA
}

\section{Actions of surgery center nurses before the systematization of perioperative nursing care}

\author{
Actitudes de los enfermeros del centro quirúrgico en la \\ sistematización de la asistencia de enfermería perioperatoria \\ Elaine Ribeiro', Keny Michelly Camargos Ferraz', Erika Christiane Marocco Duran ${ }^{3}$
}

RESUMO: Introdução: A Sistematização da Assistência de Enfermagem Perioperatória (SAEP) trata-se de um valioso instrumento para assistência do paciente de forma integralizada, contínua, segura e humanizada pela enfermagem, sendo composta por cinco fases: visita pré-operatória de enfermagem, planejamento, implementação, avaliação e reformulação da assistência a ser planejada. Objetivo: Descrever as atitudes dos enfermeiros relacionadas à SAEP em um centro cirúrgico (CC) de um hospital no interior paulista. Métodos: Estudo transversal, descritivo. Resultados: Evidenciou-se pouco conhecimento e contato dos enfermeiros com a SAEP; as atitudes dos enfermeiros sobre esta obteve escore 89,55; a maioria dos adjetivos recebeu escore $\geq 5,5$; quanto maior o tempo trabalhado na instituição, maior o contato com a SAEP. Conclusão: É notório que a implementação da SAEP é um desafio para o enfermeiro cirúrgico, mesmo se tratando de uma ferramenta para tornar a assistência de enfermagem individualizada e eficaz, minimizando riscos e complicações pós-operatórias. Palavras-chave: Pesquisa em enfermagem. Enfermagem perioperatória. Cuidados de enfermagem. Atitude do pessoal de saúde.

ABSTRACT: Introduction: The Perioperative Nursing Care Systematization (PNCS) is a valuable instrument for patient's care in a full, continuous, safe and humanized manner by the nursing team, which is comprised of five phases: nursing preoperative visit, planning, implementation, evaluation and reformulation of the care to be planned. Objective: To describe the nurses' actions regarding PNCS in a surgery center (SC) from a hospital in the countryside of São Paulo. Methods: Cross-sectional and descriptive study. Results: We observed little knowledge and contact of the nurses with the PNCS; the actions of the nurses regarding it obtained a score of 89.55 ; most of the adjectives scored $\geq 5.5$; the higher the time worked in the institution, the higher the contact with the PNCS. Conclusion: The implementation of the PNCS is notable and a challenge for surgery nurses, even if it is a tool to make nursing care individualized and effective; therefore, minimizing postoperative risks and complications. Keywords: Nursing research. Perioperative nursing. Nursing care. Actions of health personnel.

RESUMEN: Introducción: La Sistematización de la Asistencia de Enfermería Perioperatoria (SAEP) se trata de un valioso instrumento para la asistencia del paciente de modo integrado, continuo, seguro y humanizado por el equipo de enfermería, y es compuesta de cinco fases: visita preoperatoria de la enfermería, planeo, implementación, evaluación y reformulación de la asistencia a planificarse. Objetivo: Describir las actitudes de los enfermeros relacionadas a la SAEP en un centro quirúrgico (CQ) de un hospital en el interior de São Paulo, Brasil. Métodos: Estudio trasversal y descriptivo. Resultados: Se observaron poco conocimiento y contacto de los enfermeros con la SAEP; las actitudes de los enfermeros sobre la SAEP obtuvieron un escore de 89,55; la mayoría de los adjetivos recibió un escore $\geq 5,5$; y cuanto mayor el tiempo trabajado en la institución, mayor el contacto con la SAEP. Conclusión: Es notorio que la implementación de la SAEP es un desafío para el enfermero quirúrgico, aún que se trate de una herramienta para tornar la asistencia de la enfermería individualizada y eficaz, minimizándose los riesgos y las complicaciones posoperatorias. Palabras clave: Investigación en enfermería. Enfermería perioperatoria. Atención de enfermería. Actitud del personal de salud.

Doutoranda no Programa de Pós-Graduação de Enfermagem da Universidade Estadual de Campinas (UNICAMP) e coordenadora e docente do curso de Pós-Graduação em Centro Cirúrgico e Centro de Materiais e Esterilização do Centro Universitário Herminio Ometto (UNIARARAS) e docente de graduação em Enfermagem da Instituição de Ensino São Francisco (IESF) e do Instituto de Ensino Superior de Itapira (IESI) - Itapira (SP), Brasil. E-mail: enf.elaine.ribeiro@gmail.com

Rua Dionisia Rocha Serra, 45 - São Vicente - CEP: 13974-615 - Itapira (SP), Brasil.

Enfermeira pela Faculdade de Enfermagem da UNICAMP - Campinas (SP), Brasil.

${ }^{3}$ Enfermeira doutora, coordenadora e docente do curso de graduação em Enfermagem da UNICAMP - Campinas (SP), Brasil.

Recebido: 19 abr. 2017 - Aprovado: 30 ago. 2017

DOl: $10.5327 / Z 1414-4425201700040005$ 


\section{INTRODUÇÃO}

O centro cirúrgico (CC) é um setor isolado e de grande complexidade dentro do contexto hospitalar. Muito dinâmico, estressante e hostil, apresenta um ambiente físico frio e fechado, o que estimula o silêncio e o distanciamento entre a equipe multidisciplinar e o paciente, transformando o cuidado em um trabalho mecânico ${ }^{1}$.

Diante desse contexto, no que se refere à assistência direta, a enfermagem enriquece e estabelece seu corpo de conhecimento próprio em suas diversas áreas a partir de uma metodologia, que se denomina processo de enfermagem (PE). Trata-se de um instrumento sistemático e humanizado utilizado para nortear o cuidado profissional de enfermagem, constituído por cinco etapas: histórico de enfermagem, diagnóstico de enfermagem, planejamento, implementação e avaliação ${ }^{2,3}$.

A operacionalização do PE acontece quando se implementa a Sistematização da Assistência de Enfermagem (SAE), que torna mais eficiente o processo de trabalho e é a estrutura que norteia o cuidar na enfermagem. No contexto perioperatório, o PE é denominado Sistematização da Assistência de Enfermagem Perioperatória (SAEP) ${ }^{4}$.

Assim, a SAEP é uma valiosa ferramenta para que o paciente seja assistido de forma integralizada, contínua, segura e humanizada pela enfermagem. Pode ainda ser compreendida como um instrumento metodológico que sistematiza a prática e proporciona percepção, interpretação e antecipação das respostas individuais às alterações de saúde. Também promove intervenção adequada, planejada e fundamentada dos problemas identificados no paciente no período perioperatório, assim como a avaliação dos resultados ${ }^{5}$.

É constituída por cinco fases, a saber:

- visita pré-operatória de enfermagem;

- planejamento da assistência perioperatória;

- implementação da assistência;

- avaliação da assistência (por meio da visita pós-operatória de enfermagem); e

- reformulação da assistência a ser planejada (segundo resultados obtidos e solução de situações não desejadas ou eventos adversos) ${ }^{1}$.

As visitas pré e pós-operatória são ferramentas que qualificam a assistência prestada ao indivíduo e à sua família no período perioperatório ${ }^{6}$.

A maioria dos profissionais acredita que a SAEP seja uma prática indispensável ao atendimento de qualidade para os pacientes, porém os mesmos ainda enfrentam algumas dificuldades para implantá-la $a^{7,8}$.

Estudos apresentam ${ }^{9,10}$ como dificuldades para implantação da SAEP:

- a não capacitação da equipe para execução do processo de enfermagem;

- falta de domínio no exame físico e na interação da equipe;

- falta de um protocolo no hospital que determine a sua realização;

- estrutura organizacional;

- funções administrativa e assistencial concomitante;

- horário de internação;

- escassez de recursos humanos;

- falta de formulário específico para a visita;

- excesso de rotinas nas unidades;

- falta de planejamento;

- mapa cirúrgico não confiável e falta de prioridade à visita pré-operatória.

Essas dificuldades aumentam ainda mais quando a administração das unidades de saúde não compreende a importância da atuação do enfermeiro na assistência ao paciente cirúrgico durante o período perioperatório, proporcionando um desvio da sua função assistencial para um papel gerencial. A literatura aponta, ainda, a falta de tempo e a sobrecarga de atividades como os principais desafios à implementação da SAEP $^{6,9,10}$.

Acredita-se, diante da literatura consultada $a^{5-8}$, que a qualidade da assistência de enfermagem prestada no período perioperatório interfere diretamente nos resultados do procedimento cirúrgico realizado. Assim, por meio de estudos dessa natureza, busca-se compreender a atuação do enfermeiro nessa unidade, destacando sua importância e envolvimento no processo de melhoria da qualidade do atendimento.

Dessa forma, as atitudes que os enfermeiros apresentam no CC figuram como uma função significativa na execução de conceitos, visto que contribuem para impulsionar a realização de comportamentos correlacionados ${ }^{11}$. Profissionais cujas atitudes são favoráveis ao PE têm facilidade para se empenhar nas mudanças requeridas na implantação e implementação da SAEP, ao contrário daqueles cujas atitudes são desfavoráveis e que, provavelmente, terão maior dificuldade de adaptação ${ }^{12}$.

Estudos apontam ${ }^{12,13}$ alguns fatores que podem contribuir para diferentes posicionamentos perante a utilização do PE, destacando: experiência na prática clínica de enfermagem; educação formal; e expectativa dos profissionais sobre si mesmos. 
É valido destacar ainda outro estudo ${ }^{14}$ que avaliou as atitudes dos enfermeiros sobre o PE por meio da tradução e da adaptação transcultural do instrumento denominado Positions on Nursing Diagnosis $^{15}$, desenvolvido originalmente com o objetivo de analisar as atitudes de enfermeiros perante o conceito de diagnóstico de enfermagem, uma das etapas do PE. Nesse estudo ${ }^{15}$, houve a substituição do conceito de diagnóstico de enfermagem (DE) pelo conceito de PE e o instrumento passou a ser denominado de posições sobre o processo de enfermagem (PPE).

A utilização da SAEP auxiliará o paciente e sua família a compreenderem e se prepararem para o tratamento anestésico-cirúrgico, diminuindo ao máximo os riscos decorrentes da utilização dos materiais e equipamentos necessários para os procedimentos. Também irá prever, prover e controlar os recursos materiais e humanos, reduzindo ao máximo os riscos inerentes aos ambientes do CC e da sala de recuperação pós-operatória ${ }^{5}$. Tendo isso em vista, torna-se imprescindível a investigação dos fatores que podem interferir na implantação e manutenção da SAEP na prática clínica do enfermeiro - sejam eles relativos ao ambiente institucional ou aos próprios profissionais de enfermagem. Tal fato conduz ao questionamento sobre as atitudes dos enfermeiros quanto à relevância da SAEP, bem como sobre as dificuldades que enfrentam para empregá-la em suas atividades diárias.

Objetiva-se com este estudo descrever as atitudes dos enfermeiros relacionadas à SAEP na unidade de CC de um hospital público de ensino no interior do estado de São Paulo.

\section{MÉTODOS}

Trata-se de um estudo descritivo, de delineamento transversal, desenvolvido junto aos enfermeiros do CC Geral de um hospital público universitário no interior paulista, cujo objetivo foi caracterizar a população em seu perfil sociodemográfico e de trabalho; descrever a disposição dos enfermeiros sobre a implementação da SAEP, além de analisar associações entre atitudes relacionadas a essa sistematização e variáveis pesquisadas (sexo, idade, cargo de "chefia", realização de pós-graduação, atividade principal no ambiente de trabalho, satisfação com o local de trabalho, satisfação com a carreira, grau de conhecimento e contato com a SAEP, e tempo de formação).

O CC apresenta 16 salas cirúrgicas, sendo 4 de urgência e emergência, 1 sala de recuperação pós-anestésica (com 6 leitos) e 1 unidade de terapia anestésica.

A amostra foi composta por 20 enfermeiros que responderam, no local de trabalho, em março de 2016, os questionários autoaplicáveis.
Foram elegíveis para participar do estudo todos os enfermeiros que atuavam no CC no período da pesquisa, sendo excluídos três profissionais por não terem preenchido o instrumento de forma correta.

Foram utilizados os seguintes parâmetros:

- caracterização da população: dados pessoais e do trabalho;

- grau de conhecimento sobre a SAEP: para cada pergunta, o participante tinha que escolher um dos quatro itens oferecidos na escala tipo Likert (nenhum, pouco, moderado ou muito), sendo que quanto maior o escore final, maior o seu conhecimento sobre SAEP;

- grau de contato com a SAEP: o contato (nos últimos três anos) foi estimado de acordo com cinco itens - atividades de leitura sobre o tema, participação em aulas ou cursos, participação em eventos específicos sobre SAEP, uso na prática clínica e realização de pesquisa sobre o assunto (para cada um desses itens, a escala de resposta foi: nada, pouco, moderado ou muito; e, quanto maior o escore, mais intenso foi considerado o contato com a SAEP);

- posição do enfermeiro sobre SAEP: para esse item, foi usado um questionário autoaplicável com objetivo de medir as atitudes dos enfermeiros sobre a SAEP, por meio do diferencial semântico para mensurá-las, sendo solicitado ao respondente que pontuasse como se sentia em relação à SAEP mediante a atribuição de 20 duplas de adjetivos. Cada dupla de adjetivos foi separada por uma escala de sete pontos.

As ferramentas de avaliação dos parâmetros de grau de conhecimento, de contato e posições, originariamente, foram desenvolvidas para o PE; entretanto, pelo fato de a SAEP apresentar as fases adaptadas para o CC, puderam ser utilizadas neste contexto.

Foi realizada análise estatística descritiva. As possíveis correlações entre a variável "idade" e as variáveis relacionadas aos escores do SAEP foram investigadas por meio do coeficiente de correlação de Pearson.

As correlações entre a variável "tempo de formado" e as variáveis relacionadas aos escores da SAEP foram avaliadas por meio do coeficiente de correlação de Sperman, pois a variável tempo de formado não apresentou distribuição normal.

Tais coeficientes variam de -1 a 1 , sendo que os valores mais próximos de - 1 indicam uma relação negativa ou inversa entre as variáveis; os valores próximos a 1 indicam uma relação positiva entre elas; e valores próximos a 0 , ausência de correlação. 
Com relação à intensidade da correlação entre as variáveis, a literatura ${ }^{16}$ classifica esse coeficiente em: fraca $(0,10 \mathrm{a}$ $0,29)$, moderada $(0,30$ a 0,49$)$ e forte (maior ou igual a 0,50$)$. Para todas as análises, foi considerado um nível de significância igual a 5\% e utilizado o software estatístico Statistical Analysis System (SAS) versão 9.4.

O estudo foi aprovado pelo Comitê de Ética sob o parecer $\mathrm{n}^{\circ}$ 1.376.228, em que todos os participantes assinaram o termo de consentimento livre e esclarecido (TCLE).

\section{RESULTADOS}

Dos 20 enfermeiros que participaram do estudo, $95 \%(n=19)$ eram do sexo feminino, com média de 45 anos (desvio padrão$\mathrm{DP}=7,47$ ), variando de 30 a 55 anos. Os profissionais estavam, em média, há 18 anos na instituição e trabalhavam 33 horas semanais. Do total de enfermeiros, $65 \%(\mathrm{n}=13)$ referiram ter cursado pós-graduação lato sensu (aprimoramento ou especialização), $15 \%(n=3)$ referiram ter cursado mestrado e $10 \%(n=2)$ haviam cursado doutorado.

Referente aos cargos, $80 \%(\mathrm{n}=16)$ dos profissionais exerciam o cargo de enfermeiro assistencial do CC; $10 \%(\mathrm{n}=2)$, cargo de supervisor; e $10 \%(n=2)$, cargo de diretor. Com relação ao turno, $30 \%(\mathrm{n}=6)$ trabalhavam no período matutino, $35 \%(\mathrm{n}=7)$ no vespertino, $20 \%(\mathrm{n}=4)$ no diurno e $15 \%(\mathrm{n}=3)$ no noturno.

Trabalhavam na unidade de preferência $85 \%(\mathrm{n}=17)$ dos profissionais; $10 \%$ ( $\mathrm{n}=9$ ) não tinham preferência pelo setor; e $5 \%(\mathrm{n}=1)$ tinham preferência por outro setor, porém não conseguiram transferência. A totalidade dos profissionais apresentava vínculo empregatício apenas com o hospital do estudo.

No que se refere à satisfação no trabalho, $55 \%(\mathrm{n}=11)$ dos profissionais apresentaram-se moderadamente satisfeitos, $35 \%$ $(\mathrm{n}=7)$ totalmente satisfeitos e $10 \%(\mathrm{n}=2)$ pouco satisfeitos.

Quanto às atividades exercidas no $\mathrm{CC}, 30 \%(\mathrm{n}=6)$ dos profissionais referiram atuar no planejamento de cuidados, $25 \%$ $(\mathrm{n}=5)$ no cuidado direto, $15 \%(\mathrm{n}=3)$ em atividades administrativas, $15 \%(\mathrm{n}=3)$ no planejamento de cuidados e atividades administrativas, $10 \%(\mathrm{n}=2)$ no cuidado direto e planejamento de cuidados e $5 \%(n=1)$ no planejamento, cuidados e outras atividades.

O conhecimento acerca da SAEP e de suas etapas foi avaliado por meio de um instrumento com cinco itens. $\mathrm{O}$ escore do instrumento variou de 4 a 20; quanto maior o escore, maior o grau de conhecimento. O escore total desse conhecimento variou de 7 a 15, com média de 10,05, mediana de 10 e $\mathrm{DP}=1,99$.

Do total de enfermeiros, $65 \%(n=13)$ responderam ter conhecimento moderado sobre a SAEP no geral. Quanto às etapas da SAEP, a maioria considerou ter conhecimento moderado no que diz respeito a: entrevista/exame físico (75\% / 15), diagnóstico de enfermagem (70\% / 14), prescrição de enfermagem $(65 \% / 13)$ e evolução de enfermagem $(70 \% / 14)$.

O grau de contato com atividades relacionadas à SAEP também foi avaliado por meio de um instrumento com cinco itens, com escore variando de 4 a 20; quanto maior o escore, maior o grau de contato com a SAEP. O escore total sobre o grau de contato com atividades relacionadas à SAEP variou de 1 a 11, com média de 4,6, mediana de 4,5 e DP=2,66.

De acordo com os questionários avaliados:

- $50 \%(\mathrm{n}=10)$ da amostra consideraram realizar pouca leitura sobre a SAEP;

- $75 \%(\mathrm{n}=15)$ referiram ter pouca participação em aulas e cursos sobre a SAEP;

- $90 \%(\mathrm{n}=18)$ referiram ter pouca ou nenhuma participação em eventos relacionados;

- $70 \%(n=14)$ relataram utilizar pouco ou em nenhum momento a SAEP na parte clínica; e

- $55 \%(\mathrm{n}=11)$ não realizaram nenhum tipo de pesquisa sobre o tema.

As atitudes dos enfermeiros sobre a SAEP foram avaliadas com o PPE. O escore desse instrumento varia de 20 a 140; quanto maior o escore, mais favorável a disposição do enfermeiro com relação à SAEP. O escore total do PPE variou de 53 a 120, com média de 89,55, mediana de 93 e DP=17,58.

Os itens que apresentaram escores médios $\leq 4,5$ foram os dos adjetivos ambíguo/claro e fácil/difícil. Os itens que apresentaram escores médios $\geq 5,5$ foram os dos adjetivos não significativo/significativo, valioso/sem valor, negativo/positivo, bobo/inteligente, inválido/válido, significante/insignificante, relevante/irrelevante, não compensador/ compensador, conveniente/inconveniente, aceitável/inaceitável, ruim/ bom e sem importância/importante.

Apesar de a maioria dos adjetivos apresentar escores médios $\geq 5,5$, alguns mantiveram-se neutros: agradável/ desagradável, forte/fraco, confortável/desconfortável, não realista/realista, facilitador/dificultador e criativo/ rotineiro.

Foram realizadas correlações entre as variáveis idade, tempo, escore de conhecimento acerca da SAEP, escore de contato com a SAEP e escore no PPE. Não foram evidenciadas 
correlações estatísticas entre idade e os instrumentos da SAEP (Tabela 1).

A Tabela 2 apresenta as correlações entre o tempo de atuação na unidade e os instrumentos da SAEP, apresentando correlação estatisticamente significativa entre esta variável e o escore de contato, ou seja, quanto maior o tempo trabalhado na instituição, maior o contato com a SAEP.

\section{DISCUSSÃO}

O sexo feminino foi predominante nessa pesquisa, o que condiz com uma ideologia socialmente construída relativa ao exercício da enfermagem. Ainda há uma predisposição em relacionar o cuidar da profissão como algo maternal ou como uma tarefa da mulher, pois envolve sensibilidade, carinho e afetuosidade, que são considerados atributos femininos ${ }^{12}$.

Um artigo identificou dez motivos pelos quais os profissionais buscam cursos de pós-graduação lato sensu: aproximação com a temática que mais gostam; a necessidade do conhecimento; exigência do mercado de trabalho; retorno do investimento financeiro; exigência da titulação pelos concursos públicos; influência de terceiros na escolha do curso; linha de progresso para o stricto sensu; exigência pessoal pautada

Tabela 1. Correlações entre idade e escore de conhecimento da Sistematização da Assistência de Enfermagem Perioperatória (SAEP), escore de contato com a SAEP e escore de Posições sobre o Processo de Enfermagem (PPE). Campinas, 2016.

\begin{tabular}{|c|c|c|c|}
\hline & $\begin{array}{c}\text { Escore } \\
\text { conhecimento }\end{array}$ & $\begin{array}{c}\text { Escore } \\
\text { contato }\end{array}$ & $\begin{array}{c}\text { Escore } \\
\text { PPE }\end{array}$ \\
\hline \multirow{2}{*}{ Idade } & $0,0097^{\mathrm{a}}$ & 0,4220 & 0,1343 \\
\hline & $0,9676^{\mathrm{b}}$ & 0,0639 & 0,5724 \\
\hline
\end{tabular}

${ }^{a}$ Coeficiente de correlação de Pearson; ${ }^{b}$ valor $p$.

Tabela 2. Correlações entre o tempo de atuação na unidade e escore de conhecimento da Sistematização da Assistência de Enfermagem Perioperatória (SAEP), escore de contato com a SAEP e escore de Posições sobre o Processo de Enfermagem (PPE). Campinas, 2016.

\begin{tabular}{|c|c|c|c|}
\hline & $\begin{array}{c}\text { Escore } \\
\text { conhecimento }\end{array}$ & $\begin{array}{c}\text { Escore } \\
\text { contato }\end{array}$ & $\begin{array}{c}\text { Escore } \\
\text { PPE }\end{array}$ \\
\hline \multirow{2}{*}{ Tempo } & $-0,1156^{\mathrm{a}}$ & 0,4602 & 0,1188 \\
\hline & $0,6275^{\mathrm{b}}$ & 0,0412 & 0,6178 \\
\hline
\end{tabular}

${ }^{a}$ Coeficiente de Spearman; ${ }^{b}$ valor $p$. pela necessidade vivenciada na prática; saber trabalhar em qualquer área; necessidade de adequação de horários ${ }^{11}$.

Em contrapartida, a pós-graduação stricto sensu tem sido imprescindível para o progresso da ciência, da tecnologia e da inovação, o que impulsiona a transformação econômica e social na qual a enfermagem brasileira transfere seu conhecimento científico para a prática profissional, melhorando o cuidado e o ensino em saúde ${ }^{17}$.

Chamou a atenção o pequeno número de profissionais que se declarou satisfeito com a profissão. A satisfação em trabalhar no local de preferência é um fator protetor contra a Síndrome de Burnout, uma vez que o ambiente hospitalar é insalubre, penoso, perigoso e propício ao adoecimento. Quanto menor a satisfação com o local de trabalho, maior é a probabilidade do desenvolvimento de exaustão emocional ${ }^{18}$.

Neste estudo, o conhecimento sobre a SAEP apresentado pela amostra foi moderado, assim como sobre todas as suas etapas (entrevista/exame físico, diagnóstico de enfermagem, prescrição de enfermagem e evolução de enfermagem). No hospital em questão, a única etapa realizada da SAEP é a visita pré-operatória.

A maioria dos enfermeiros referiu realizar pouca leitura sobre a SAEP, ter pouca ou nenhuma participação em aulas ou cursos sobre o tema, pouca ou nenhuma participação em eventos relacionados, usar em poucos ou em nenhum momento a SAEP na atuação clínica e não ter realizado qualquer tipo de pesquisa relacionada.

Esse resultado corrobora COM outro achado ${ }^{14}$ sobre a percepção dos enfermeiros quanto ao significado da SAE, o qual mostrou que houve um déficit sobre o conhecimento dos enfermeiros para sua implementação.

Dessa forma, para uma assistência de qualidade é preciso que os profissionais sejam qualificados, e a educação continuada é uma oportunidade para o aprimoramento 5 .

Destaca-se, ainda, que para a efetiva implementação da SAEP se faz necessário que o enfermeiro tenha conhecimento sobre a aplicabilidade dos diagnósticos de enfermagem, bem como sobre suas intervenções e resultados junto aos pacientes, utilizando-se da Taxonomia II da Nanda- ${ }^{19}$, aliada à classificação das intervenções de enfermagem (NIC) e à classificação de resultados de enfermagem (NOC ${ }^{20}$.

Referente à pontuação média nos itens do $\mathrm{PPE}$, a menor média foi direcionada à dupla de adjetivos fácil/ difícil $(3,35)$. A literatura propõe analisar os itens com escore $\geq 5,5$ como fortemente favoráveis; e aqueles com escore médio $\leq 4,5$ como mais desfavoráveis ${ }^{15}$. 
Neste estudo, o maior escore médio foi observado no item sem importância/importante $(6,2)$ classificado como fortemente favorável $(\geq 5,5)$, semelhante a outros trabalhos ${ }^{14}$.

Já com relação aos pontos positivos da SAEP, foram indicados pelos adjetivos: significativo, valioso, positivo, inteligente, válido, significante, relevante, recompensador, conveniente, aceitável, bom e importante.

Houve correlação somente entre o maior tempo de trabalho na instituição e o contato com a SAEP. Esse achado pode sugerir que trabalhar em um hospital universitário propicia o contato com conhecimento científico, sem, no entanto, garantir a utilização deste.

Essa realidade nos permite inferir que aqueles enfermeiros que não utilizam o DE, ou fazem uso dele sem preocupação com a sua precisão, mantêm a invisibilidade de seu papel como diagnosticador ${ }^{19}$.

Nesse contexto, acredita-se, ainda, que o enfermeiro precisa dedicar-se à realização da SAEP, tendo em vista que a prática em saúde no CC demanda estudos de intervenção para que os conceitos já desenvolvidos possam ser validados no cotidiano da assistência, explicitando suas contradições e possibilidades, as quais representam um desafio para o enfermeiro, possível e essencial.

Como limitações para construção deste estudo destacam-se a dificuldade dos enfermeiros da unidade do referido CC em disponibilizar tempo para participar da pesquisa e a escassez de referencial teórico para a discussão sobre o tema proposto.

\section{CONCLUSÃO}

Observou-se que quanto mais tempo de trabalho o enfermeiro apresentava na instituição, maior o contato com a SAEP; porém, há ainda fragilidades na sua implementação.

Um dos fatores que demonstram a baixa adesão ao uso da SAEP, neste estudo, refere-se ao fato de que apenas uma etapa é realizada durante o período perioperatório, o que pode ser atribuído à pouca procura de atualizações referentes ao tema, tanto por desmotivação do próprio profissional quanto pela falta de estímulo da instituição.

Evidenciou-se, ainda, que a maioria dos enfermeiros participantes desta pesquisa refere realizar pouca leitura sobre a SAEP, o que dificulta sua aplicabilidade prática.

Acredita-se que a baixa produção científica em relação à SAEP pode estar relacionada ao fato de os próprios profissionais não se interessarem em pesquisar o assunto, como foi apresentado neste estudo.

Conclui-se que a SAEP é fundamental para uma assistência de qualidade; entretanto, não é um processo fácil para o enfermeiro e sua equipe, requerendo que os profissionais tenham iniciativa e pró-atividade para superar os obstáculos evidenciados, tendo em vista que a prática em saúde no CC demanda estudos de intervenção para que os conceitos já desenvolvidos possam ser validados no cotidiano da assistência.

\section{REFERÊNCIAS}

1. Silva DC, Alvim NAT. Ambiente do Centro Cirúrgico e os elementos que o integram: implicações para os cuidados de enfermagem. Rev Bras Enferm [Internet]. 2010 [citado em 12 jun. 2016];63(3):427-34. Disponível em: http://www.scielo.br/pdf/reben/v63n3/a13v63n3.pdf

2. Silva MB, Ceretta RSR, Zuse CL, Fontana RT. Diagnósticos de enfermagem na percepção de graduandos em enfermagem. Rev Pesq Cuid Fundam [Internet]. 2012 [citado em 12 jun. 2016];4(2):2964-72. Disponível em: http://www.seer.unirio.br/ index.php/cuidadofundamental/article/view/1843

3. Garcia TR, Nóbrega MML. Processo de enfermagem: da teoria à prática assistencial e de pesquisa. Esc Anna Nery Rev Enferm [Internet]. 2009 [citado em 12 jun. 2016];13(1):188-93. Disponível em: http:// www.scielo.br/pdf/ean/v13n1/v13n1a26.pdf

4. Figueiredo MED, Santos SR, Oliveira AMM, Leite KNS, Morais JMD, Duarte ACP. Systematization of nursing care: perceptions of nurses of a teaching school. Rev Enferm UFPE [Internet]. 2013 [citado em 12 jun. 2016];7(12):6981-8. Disponível em: http://www. revista.ufpe.br/revistaenfermagem/index.php/revista/article/ view/4828/pdf_4218
5. Sociedade Brasileira de Enfermeiros de Centro Cirúrgico, Recuperação Anestésica e Centro de Material e Esterilização (SOBECC). Centro Cirúrgico, Recuperação Anestésica, Centro de Material e Esterilização: Práticas Recomendadas SOBECC. 6a ed. São Paulo: SOBECC; 2013.

6. Oliveira MM, Mendonça KM. Análise da visita pré-operatória de enfermagem: revisão integrativa. Rev SOBECC. 2014;19(3):164-72.

7. Silva EGCS, Oliveira VC, Neves GBC, Guimarães TMR. O conhecimento do enfermeiro sobre a sistematização da assistência de enfermagem: da teoria à prática. Rev Esc Enferm USP [Internet]. 2011 [citado em 12 jun. 2016];45(6):1380-6. Disponível em: http://www.scielo.br/pdf/ reeusp/v45n6/v45n6a15.pdf

8. Santos MC, Rennó CSN. Indicadores de qualidade da assistência de enfermagem em centro cirúrgico: revisão integrativa da literatura. RAS. 2013;15(58):27-36.

9. Casafus KC, Dell'Acqua MCQ, Bocchi SCM. Entre o êxito e a frustração com a sistematização da assistência de enfermagem. Esc Anna Nery [Internet]. 2013 [citado em 12 jun. 2016];17(2):313-21. Disponível em: http://www.scielo.br/pdf/ean/v17n2/v17n2a16.pdf 
10. Lemos CS, Suriano MLF. Desenvolvimento de um instrumento: Metodologia de ensino para aprimoramento da prática perioperatória. Rev SOBECC. 2013;18(4):57-69.

11. Barbosa IG. Os motivos do enfermeiro para a inserção em cursos lato sensu sob olhar da complexidade. Rev Enferm. 2011;(2):276. Suplemento Actas e Comunicações da XI Conferência Iberoamericana de Educação em Enfermagem. Loures: Lusodidacta.

12.Diogo MF. Savoir-faire feminino e sua apropriação profissional pelo olhar das relações de gênero. Psicol Argum. [Internet]. 2012 [citado em 12 jun. 2016];30(71):731-43. Disponível em: http://www2.pucpr.br/reol/pb/index.php/ pa?dd1 $=7479 \& d d 99=$ view\&dd98=pb

13. Souza MFG, Santos ADB, Monteiro Al. 0 processo de enfermagem na concepção de profissionais de enfermagem de um hospital de ensino. Rev Bras Enferm [Internet]. 2013 [citado em 12 jun. 2016];66(2):167-73. Disponível em: http://www.scielo.br/scielo. php?script=sci_arttext\&pid=S0034-71672013000200003

14. Guedes ES, Turrini RNT, Sousa RMC, Baltar VT, Cruz DALM. Atitudes do pessoal de enfermagem relacionadas ao processo de enfermagem. Rev Esc Enferm USP. 2012;46(Esp):130-7.
15. Lunney M, Krenz MA. An instrument to measure attitudes toward nursing diagnosis. In: Carrol-Johnson RM, Paquette M, eds. Classification of Nursing Diagnoses Proceedings of the Tenth Conference of North American Nursing Diagnosis Association. San Diego: Lippincott; 1992. p.389-90.

16. Cohen J. Statistical power analysis for the behavioral sciences. $2^{\text {a }}$ ed. New Jersey: Lawrence Erlbaum Associates; 1988. p.75-108.

17. Scochi CGS, Munari DB, Gelbcke FL, Ferreira MA. Desafios e estratégias dos programas de pós-graduação em enfermagem para a difusão da produção científica em periódicos internacionais. Esc Anna Nery [Internet]. 2014 [citado em 12 jun. 2016];18(1):5-10. Disponível em: http://www.scielo.br/scielo. php?script=sci_arttext\&pid=S1414-81452014000100005

18. Neves VF, OliveiraAF, Alves PC. Síndrome de Burnout: impacto da satisfação no trabalho e da percepção de suporte organizacional. Psico [Internet]. 2014 [citado em 12 jun. 2016];45(1):45-54. Disponívelem: http://revistaseletronicas. pucrs.br/ojs/index.php/revistapsico/article/view/12520/11440

19. Nanda. Diagnósticos de enfermagem da Nanda: Definições e Classificação. 2015-2017. 10a ed. Porto Alegre: Artmed; 2015. 468p.

20. Johnson M. Ligações Nanda Noc-Nic: condições clínicas suporte ao raciocínio e assistência de Qualidade. Rio de Janeiro: Elsevier; 2012. 422p. 